\title{
Aflibercept treatment for neovascular AMD beyond the first year: consensus recommendations by a UK expert roundtable panel, 2017 update
}

\author{
This article was published in the following Dove Press journal: \\ Clinical Ophthalmology \\ 6 November 2017 \\ Number of times this article has been viewed
}

\section{Praveen J Patel,' Helen \\ Devonport, ${ }^{2}$ Sobha \\ Sivaprasad,' Adam H Ross, ${ }^{3}$ \\ Gavin Walters, ${ }^{4}$ Richard P \\ Gale, ${ }^{5}$ Andrew J Lotery, ${ }^{6}$ \\ Sajjad Mahmood, ${ }^{7}$ James $\mathrm{S}$ \\ Talks, ${ }^{8}$ Jackie Napier ${ }^{9}$}

'National Institute for Health Research Biomedical Research Centre at Moorfields Eye Hospital NHS Foundation Trust and UCL Institute of Ophthalmology, London, UK;

${ }^{2}$ The Ophthalmology Department, Bradford Royal Infirmary, Bradford, UK; ${ }^{3}$ The Ophthalmology Department, Bristol Eye Hospital, Bristol, UK; ${ }^{4}$ Department of Ophthalmology, Harrogate District Hospital, Harrogate, UK; ${ }^{5}$ The Ophthalmology Department, The York Hospital and Department of Health Sciences, University of York, York, UK; ${ }^{6}$ Clinical and Experimental Sciences, Faculty of Medicine, University of Southampton, Southampton, UK; ${ }^{7}$ Manchester Royal Eye Hospital, Central Manchester University Hospitals NHS Foundation Trust, Manchester Academic Health Science Centre, Manchester, UK; ${ }^{8}$ Newcastle Eye Centre, Royal Victoria Infirmary, Newcastle upon Tyne, UK; 'Medical Affairs, Bayer plc, Reading, Berkshire, UK

Correspondence: Praveen J Patel Moorfields Eye Hospital NHS

Foundation Trust, 162 City Road,

London ECIV 2PD, UK

$\mathrm{Tel}+44020725334$ II

Email praveen.patel@moorfields.nhs.uk
Abstract: National recommendations on continued administration of aflibercept solution for injection after the first year of treatment for neovascular age-related macular degeneration (nAMD) have been developed by an expert panel of UK retina specialists, based on clinician experience and treatment outcomes seen in year 2. The 2017 update reiterates that the treatment goal is to maintain or improve the macular structural and functional gains achieved in year 1 while attempting to reduce or minimize the treatment burden, recognizing the need for ongoing treatment. At the end of year 1 (ie, the decision visit at month 11), two treatment options should be considered: do not extend the treatment interval and maintain fixed 8-weekly dosing, or extend the treatment interval using a treat-and-extend regimen up to a maximum 12 weeks. Criteria for considering not extending the treatment interval are persistent macular fluid with stable vision, recurrent fluid, decrease in vision in the presence of fluid, macular hemorrhage, new choroidal neovascularization or any other sign(s) of exudative disease activity considered vision threatening in the opinion of the treating clinician. Treatment extension is recommended for eyes with a dry macula (ie, without macular fluid) and stable vision. Under both options, the treatment interval may be shortened if visual and/or anatomic outcomes deteriorate. Monitoring without treatment may be considered for eyes with a fluid-free macula for a minimum duration of 48 weeks. A patient completing one full year of monitoring without requiring injections may be considered for discharge from clinic. The treatment algorithm incorporates return to fixed 8-weekly dosing for disease reactivation during treatment extension and reinstatement of treatment for disease recurrence following discontinuation or discharge. For bilateral nAMD, either the eye requiring the more intensive treatment or the eye with the better vision, guided by local clinical practice, should determine the retreatment schedule overall.

Keywords: anti-vascular endothelial growth factor, maintenance therapy, treatment algorithm, treat-and-extend, visual acuity

\section{Introduction}

Neovascular age-related macular degeneration (nAMD) is an acute-onset, chronic progressive eye disease that affects central vision and is characterized by abnormal growth and leakage of blood vessels in the macula. Clinical features that indicate the presence of nAMD when seen within the macular area of the fundus include intraretinal, subretinal or sub-retinal pigment epithelium (RPE) hemorrhages and/or fluid with or without periretinal fibrosis in the absence of other retinal (vascular) disorders. ${ }^{1}$ The standard of care for treating nAMD is intravitreal anti-vascular endothelial growth factor 
(anti-VEGF) therapy, and continued treatment beyond 2 years may be needed to maintain control of disease activity.

Visual outcomes of anti-VEGF treatment for nAMD reported from clinical settings have often been less favorable than those observed in pivotal AMD randomized controlled trials. $^{2,3}$ Inferior visual and anatomic outcomes have been linked to undertreatment and the use of pro re nata (PRN, as-needed) dosing regimens in routine clinical practice. In the Comparison of AMD Treatments Trials follow-up study, vision gains achieved during the first 2 years with anti-VEGF treatments were lost over the next 3 years following release from the clinical trial protocol. ${ }^{4}$ There was a mean 11-letter loss from the end of year 2 to year 5 , and the final mean visual acuity (VA) was worse than that at baseline. ${ }^{4}$ Overtreatment, on the other hand, creates an unnecessary treatment burden, higher health care costs and increased risk associated with frequent injections. The timing and frequency of anti-VEGF injections after the initial year of treatment is, therefore, a crucial consideration for longer-term maintenance of visual and morphological improvements in nAMD.

A national roundtable panel of UK retina specialists met in London in May 2016 to review evidence and share experience on the use of intravitreal aflibercept therapy for nAMD. Additional review was provided by a supplementary group of retina specialists subsequent to this meeting. The overall aim was to develop practical suggestions and recommendations on administration of aflibercept for $\mathrm{nAMD}$ in the second year of treatment and beyond. These consensus recommendations supersede an earlier guideline published in 2015, to which the current authors all contributed, which was based on early initial experience with aflibercept in nAMD. ${ }^{5}$

These 2017 updated recommendations focus clinician attention at the end of year 1 on determining the most appropriate approach to dosing frequency for the second year and later of aflibercept treatment: extend or do not extend the treatment interval, based on protocol-driven criteria. The panel noted capacity and service constraints in providing monitoring clinics for patients with inactive disease and stable vision. Moreover, many patients with macular (intraretinal/subretinal) fluid seen on optical coherence tomography (OCT) images may not necessarily have active neovascular disease, while patients with so-called inactive disease may sometimes experience decreased vision. Questions addressed by the panel included frequency of administration when using aflibercept, continuous fixedversus variable-dosing regimens, monitoring nonaffected second eyes for fellow eye involvement, and factors to consider indicating that treatment for nAMD may be stopped.
The proposed treatment algorithm captures return to fixed 8-weekly dosing for disease reactivation during an extension phase and the need to reinstate treatment for disease recurrence after treatment discontinuation or discharge. The recommendations are presented and discussed in this article, with a summary provided in Table 1 and the recommended algorithm for year 2 onwards detailed in Figure 1.

It is worth noting that all eyes differ in the need for repeat injections. ${ }^{6}$ While fixed dosing remains an important approach with practical advantages, more flexible treatment options from year 2 onwards are needed to take account of resource provision and patient preference.

\section{Anti-VEGF treatment posology for nAMD}

Ranibizumab and aflibercept are licensed anti-VEGF treatments indicated in adults for the treatment of nAMD as well as for visual impairment due to other common retinal diseases. In the UK, the National Institute for Health and Care Excellence (NICE) recommends both intravitreal agents as possible treatment options for people with nAMD, subject to the following baseline criteria: the best-corrected visual acuity (BCVA) is between 6/12 and 6/96, there is no permanent structural damage to the central fovea, the area affected by AMD is $\leq 12$ disc areas in size, and there is evidence of recent presumed disease progression. ${ }^{7}$ Treatment should be stopped if the patient's vision deteriorates and there are changes inside the eye that show that treatment is not working. Ranibizumab and aflibercept are accepted for use in NHS Scotland in adults with nAMD. ${ }^{8}$

The recommended dose for ranibizumab is $0.5 \mathrm{mg}$ given as a single intravitreal injection. ${ }^{9}$ Treatment is initiated with one injection per month until maximum VA is achieved and/or there are no signs of disease activity (ie, no change in VA and in other signs and symptoms of the disease under continued treatment). Initially, three or more consecutive, monthly injections may be needed. Thereafter, monitoring and treatment intervals should be determined by the clinician based on disease activity, as assessed by VA and/or anatomical parameters.

Aflibercept treatment for nAMD involves regular 8 -weekly dosing following three initial monthly doses through the initial year of therapy. ${ }^{10}$ For the next year, the treatment interval may be extended, such as with a treat-andextend dosing regimen. If visual and/or anatomic outcomes deteriorate, the treatment interval should be shortened accordingly. The full recommended posology for aflibercept in adults with nAMD is detailed in Table 2. 
Table I Summary of recommendations for aflibercept treatment of nAMD after year I, 2017 update*

Goal of treatment beyond year I

- To maintain or improve the macular structural and functional gains achieved in year I while attempting to reduce or minimize the treatment burden (visits and/or injections), recognizing the need for continued long-term treatment

Treatment decision at the end of year I (at the time of the seventh injection in year I)

- Do not extend the treatment interval in eyes that meet one or more "Criteria for considering not extending": persistent macular fluid and stable vision, recurrent fluid, decrease in vision and presence of fluid, macular hemorrhage, new CNV or any other sign or signs of exudative disease activity considered vision threatening

- Extend the treatment interval in eyes without macular fluid and stable VA, gradually by I-2-week intervals, fixing the interval at the maximal fluid-free period and up to a maximum of 12 weeks

- Applicable to both options:

- The treatment interval may be shortened if visual and/or anatomic outcomes deteriorate

- OCT assessment should be performed and VA recorded at every clinic visit post-year I; additional monitoring visits are not required between injections

Managing disease reactivation during treatment extension phase

- For eyes that meet one or more "Criteria for considering not extending":

- Return to the previous treatment interval at which the macula remained dry (with requirement for loading at the discretion of the treating clinician)

- Fix and maintain dosing at this interval for three consecutive injections before attempting to extend again

- If extension fails once again, fix and maintain dosing at the previous shorter retreatment interval

Monitoring without treatment (following treat-and-extend therapy)

- Recommended for eyes with a dry macula sustained for 48 weeks' duration or more (ie, fluid-free for three consecutive injections given at I2-week intervals and remains dry at the next I2-week interval)

Discharge protocol

- Patients completing at least I year of monitoring without treatment may be considered for discharge from clinic, subject to local commissioning arrangements

Fellow eye involvement and approach to treating bilateral disease

- For unilateral nAMD, monitor both eyes using OCT to ensure early detection of second eye involvement

- For bilateral nAMD:

- Either the eye requiring the more intensive treatment or the eye with the better vision, guided by local clinical practice, should determine the retreatment schedule overall

- Tailor the treatment interval to patient visits in order to synchronize simultaneous (ie, consecutive) same-visit treatment, if possible

Safety and other considerations

- Ensure that the benefit-risk profile of aflibercept is discussed with the patient before initiating treatment and each time the treatment regimen is altered

- Have an informed discussion with the patient to determine treatment priorities and preferences, including any limiting comorbidities

Notes: *Treatment algorithm outlined in Figure I details "Criteria for considering not extending" the treatment interval and other decision-making criteria.

Abbreviations: CNV, choroidal neovascularization; nAMD, neovascular age-related macular degeneration; OCT, optical coherence tomography; VA, visual acuity.

\section{Guideline assumptions}

While the recommendations relate to previously untreated nAMD patients, the expectation is that long-term approaches would be similar for patients switched from another antiVEGF treatment, although treatment-resistant nAMD does not fall within the scope of these presented consensus recommendations. The guidance assumes that patients have been treated according to the licensed treatment posology for aflibercept in nAMD for the first year. As most clinics are organized using weekly rather than monthly schedules, 8 -weekly and every 2 months are considered interchangeable and mean the same for practical service provision purposes. The decision on how to treat after year 1 should be made at the time of the seventh injection visit in year 1 (ie, at around week 40 or month 11), assuming that the recommended posology in the aflibercept product label has been followed.
If there is persistent macular fluid on OCT images and a limited response to aflibercept treatment, further imaging investigations should be considered to confirm whether persistent fluid is due to structural change and to evaluate for the presence of any other ocular pathology such as polypoidal choroidal vasculopathy or central serous chorioretinopathy. Possible reasons for stopping treatment will be case-dependent, but suggested criteria include poor vision (ie, worse than 6/96) and severe central structural damage such as significant fibrosis. The threshold for stopping treatment may be lower for the first-affected eye than for the second-affected eye. In all cases, the requirement for three consecutive monthly loading doses on resumption of treatment for disease recurrence or treatment intensification on disease reactivation is at the discretion of the treating clinician. Throughout treatment, other aspects of the eye 
End of year $1^{a}$

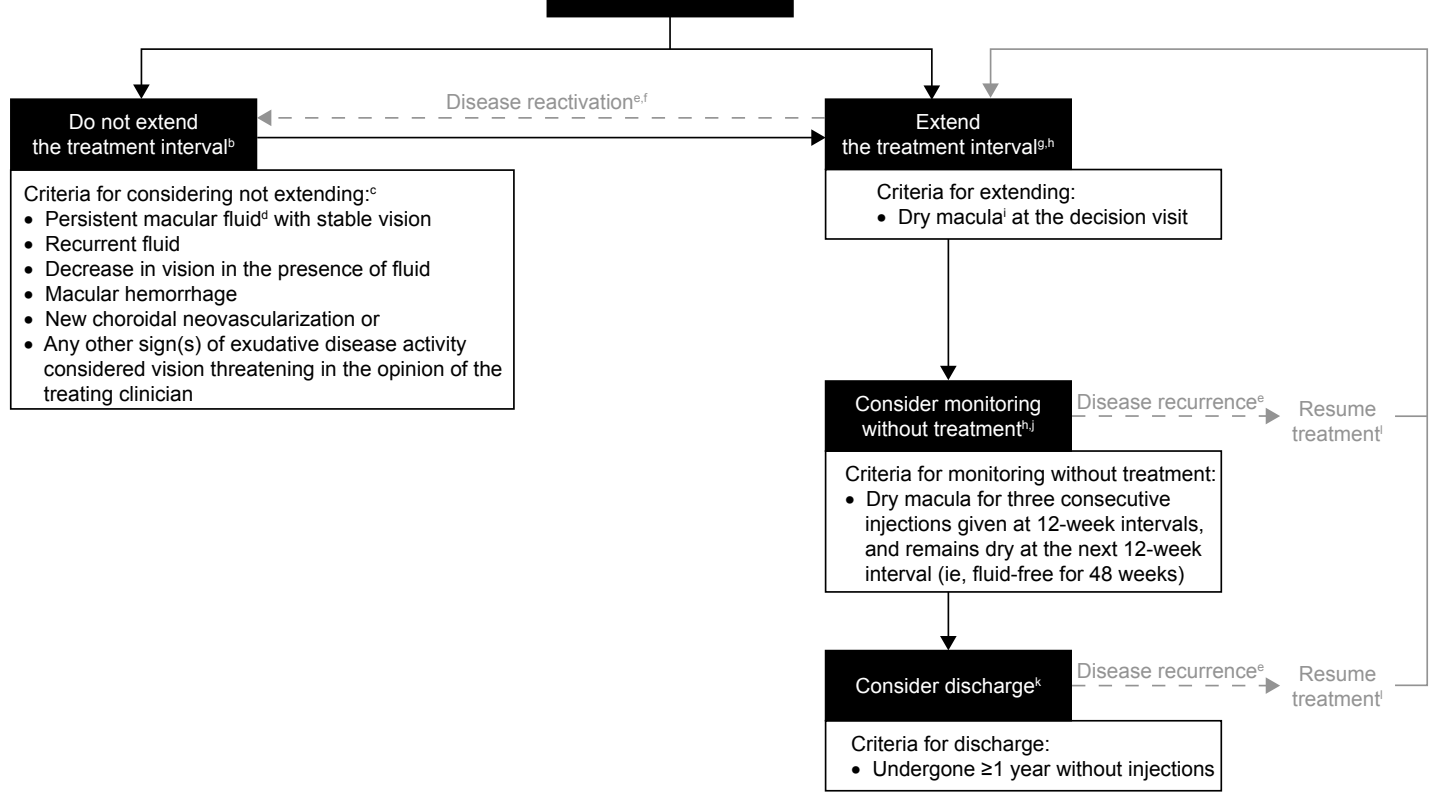

Figure I Aflibercept treatment algorithm for nAMD after year I, 2017 update.

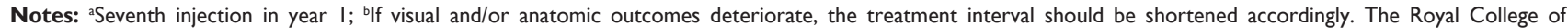
Ophthalmologists guidelines for nAMD advise that hyperactive lesions may for a short time require more intensive therapy, at the discretion of the treating clinician; 'Patients must meet at least one "Criteria for considering not extending"; ' Macular fluid refers to intraretinal/subretinal fluid; eDefined as meeting at least one "Criteria for considering not extending"; 'Return to the previous treatment interval at which the macula remained dry; ${ }^{8}$ Once the optimal dosing interval has been identified, fix the dosing at this interval for three consecutive injections before considering extending further; 'Fellow eye involvement may drive the monitoring/retreatment interval; 'In the opinion of the treating clinician; iThe next initial monitoring visit may take place 4-6 weeks later at the discretion of the treating clinician. Subsequent monitoring intervals without treatment should then be extended by 4 weeks at a time, up to a maximum of 12 weeks; ${ }^{k}$ The status of the fellow eye must be considered in any discharge assessment/decision; 'Requirement for loading is at the discretion of the treating clinician.

Abbreviation: $\mathrm{nAMD}$, neovascular age-related macular degeneration.

should be regularly monitored, such as intraocular pressure and signs of referable diabetic retinopathy in patients with diabetes.

\section{Recommendations Treatment goal}

The treatment goal in the second year and beyond is to maintain or improve the macular structural and functional gains achieved in year 1 while attempting to reduce or minimize the burden of clinic visits and injections, recognizing the need for continued long-term treatment.
The goal of a treat-and-extend dosing regimen (an example of a proactive treatment regimen) in year 2 is to determine the optimal treatment interval (ie, maximal fluidfree interval) at which patients can achieve good control of disease activity and stabilization of VA.

\section{Treatment decision on continued administration protocol for year 2}

The following options for continuation of treatment should be considered at the time of the decision visit for year 2 :

Table 2 European posology of aflibercept for nAMD

- The recommended dose is $2 \mathrm{mg}$ aflibercept, equivalent to $50 \mu \mathrm{l}$, administered by intravitreal injection

- Treatment is initiated with one injection per month for three consecutive doses, followed by one injection every 2 months

- There is no requirement for monitoring between injections

- After the first 12 months of treatment, the treatment interval may be extended, such as with a treat-and-extend dosing regimen, based on visual and/or anatomic outcomes

- In this case the treatment intervals are gradually increased to maintain stable visual and/or anatomic outcomes; however, there are insufficient data to conclude on the length of these intervals

- The treatment interval should be shortened accordingly if visual and/or anatomic outcomes deteriorate

- The schedule for monitoring should, therefore, be determined by the treating physician and may be more frequent than the schedule of injections

Notes: Eylea ${ }^{\circledR}$ (aflibercept solution for injection) [summary of product characteristics]. Leverkusen, Germany: Bayer AG; 20I7. Available from: https://www.medicines.org. uk/emc/medicine/27224..$^{10}$

Abbreviation: nAMD, neovascular age-related macular degeneration. 
- Option 1: Do not extend the treatment interval

- Criteria for considering not extending the treatment interval are persistent macular fluid with stable vision, recurrent fluid, decrease in vision in the presence of fluid, macular hemorrhage, new choroidal neovascularization (CNV) or any other sign or signs of exudative disease activity considered vision threatening in the opinion of the treating clinician.

- Option 2: Extend the treatment interval

- Treatment extension is appropriate for eyes without macular fluid on OCT images and stable VA at the end of year 1 . The treatment interval should be increased gradually by 1-2-week intervals, capping the interval at the maximal fluid-free interval and up to a maximum of 12 weeks.

During ongoing aflibercept maintenance treatment in year 2, an extension of the 8-weekly treatment interval is also recommended for:

- eyes that met at least one "Criteria for considering not extending" but then the macula became dry later;

- eyes that have experienced disease recurrence during monitoring or after discharge from clinic, have undergone retreatment and the macula then became dry;

- eyes with persistent fluid that is stable over three consecutive visits and judged to be due to long-term anatomical change or related to fibrosis rather than active $\mathrm{CNV}$.

Under both options (ie, do not extend or extend), the treatment interval may be shortened if visual and/or anatomic outcomes deteriorate. As recommended in management guidance from the Royal College of Ophthalmologists, more intensive treatment may be required in occasional cases of hyperactive lesions. ${ }^{1}$ In all cases, OCT assessment of the macula should be performed and VA recorded at every clinic visit post-year 1. Additional monitoring visits are not required between injections.

\section{Managing disease reactivation during treatment extension}

Disease reactivation refers to eyes that demonstrate one or more "Criteria for considering not extending" during a treatment extension phase, for example new macular hemorrhage. Patients who demonstrate disease reactivation during treatment extension can undergo more intensive treatment, at the discretion of the treating clinician. Treatment frequency is then set at the previous treatment interval at which the macula remained dry and fixed at this interval for three consecutive injections before attempting to extend the interval between treatments once again. If extension fails, fix and maintain treatment at the previous shorter retreatment interval (a treat-and-extend and "fix" approach) may be considered. Eyes with significant disease reactivation will require more frequent monitoring with treatment where necessary, for example in eyes with new CNV activity, symptomatic reduction in VA, extensive subretinal hemorrhage or marked morphological change on OCT findings. Fellow eye involvement may influence the decision on retreatment interval when managing disease reactivation during extension.

\section{Monitoring without treatment (following a treat-and-extend or monitor and extend approach)}

A trial of monitoring without treatment may be suitable for a proportion of nAMD eyes treated with aflibercept but this is not a recommended option at the end of year 1. Following treatment using a treat-and-extend approach in year 2, monitoring without treatment should be considered for eyes that have been fluid-free for a duration of 48 weeks (ie, eyes with a macula that has remained dry for three consecutive injections given at 12-week intervals and that remains dry at the next 12-week interval). No injection is administered at the visit occurring at the time of the fourth 12 -week interval. The next initial monitoring visit may take place 4-6 weeks later, at the discretion of the treating clinician. Subsequent monitoring intervals without treatment should then be extended by 4 weeks at a time, up to a maximum of 12 weeks. As for other decision steps, fellow eye involvement may be considered when determining an appropriate monitoring interval.

Disease recurrence refers to an eye that meets one or more "Criteria for considering not extending" the treatment interval. In eyes that demonstrate disease recurrence during monitoring without treatment, aflibercept therapy should be resumed and dose intensification considered. After one fluidfree treatment visit, extension of the treatment interval can be attempted once more. For eyes with mild disease recurrence, for example, occurring $>3$ months after entering monitoring and which became dry after only one injection, monitoring without treatment might then be resumed.

\section{Discharge protocol}

It is recognized that full discharge from the eye clinic is rare and not possible for many patients who have chronic nAMD because of the need for ongoing follow-up and care. However, patients completing at least 1 year of monitoring without requiring an injection may be considered for discharge from clinic, subject to local commissioning arrangements. When eligible for possible discharge, the patient should be seen by a specialist to allow for a full and informed discussion. The status of the fellow eye must be considered in any 
discharge assessment/decision. If discharged, patients should be reviewed by an optometrist at regular intervals, with frequency of review based on risk of vision loss, patient preference and locally agreed pathways (eg, quarterly or every 6 months). Virtual clinic models may be implemented to refine patient pathways and improve service capacity, allowing separate online specialist or consultant-supervised review of acquired OCT images. In cases of disease recurrence after discharge from clinic, treatment should only be reinstated if the patient still meets baseline eligibility criteria detailed in the NICE technology appraisal for aflibercept in nAMD. ${ }^{7}$ A fast-track referral process needs to be in place to ensure access to prompt treatment. When resuming treatment, dose intensification may be considered. After one dry visit, extension of the treatment interval can be attempted once more.

\section{Fellow eye involvement and treatment of bilateral disease}

For unilateral nAMD, both eyes should be monitored using OCT to ensure that second eye involvement is detected early. Once a patient has been diagnosed as having nAMD in one eye, the risk of developing advanced AMD in the second or fellow eye is high. The Age-Related Eye Disease Study reported that participants with advanced AMD in one eye or vision loss due to nonadvanced AMD in one eye had a $43 \%$ expected probability of progression to advanced AMD in the second eye at 5 years. ${ }^{11}$ It is, therefore, important to closely monitor both eyes using OCT to ensure the best visual prognosis.

If a patient is receiving bilateral intravitreal anti-VEGF therapy, either the eye requiring the more intensive treatment or the eye with the better vision (ie, higher VA letter score), guided by local clinical practice, should determine the retreatment schedule overall. Also, the treatment interval should be tailored to patient visits in order to synchronize simultaneous treatment for both eyes during one visit, if possible. If visual function is similar between the two eyes (VA difference $\leq 5$ Early Treatment Diabetic Retinopathy Study [ETDRS] letters), retreatment is based on assessment of the eye with the more active exudative disease. It is preferable to administer the same anti-VEGF agent in both eyes, and the use of separate vials from different batches with different lot numbers is advised to reduce the risk of bilateral endophthalmitis.

\section{Safety and other considerations}

The benefit-risk profile of aflibercept should be discussed with the patient before initiating treatment and each time the treatment regimen is altered. The patient should be informed that there is a small risk of ocular adverse events associated with treatment, mostly related to the injection procedure. An informed discussion with the patient will help to determine treatment priorities and preferences, including any limiting comorbidities. Some patients, for example those who wish to retain driving vision standard, may be willing to accept more frequent injections than others, and some may prefer a trial of monitoring without treatment.

\section{Discussion}

The clinical recommendations presented are based on consideration of real-world treatment outcomes from multicenter clinical studies and service audits, augmented by growing practitioner experience with use of aflibercept for the treatment of nAMD. When reviewing dosing frequency and treatment approaches with anti-VEGF maintenance therapy for $n A M D$, it is worth recalling relevant findings from pivotal randomized controlled trials.

The VIEW Phase III studies, the largest controlled trials of anti-VEGF agents in AMD ever performed, demonstrated that 8 -weekly $2 \mathrm{mg}$ aflibercept dosing after three initial monthly doses $(2 \mathrm{q} 8)$ provided gains in VA that were equivalent to those achieved with monthly ranibizumab over 1 year. ${ }^{12}$ Despite fewer injections being required, aflibercept was as effective as ranibizumab in increasing VA and reducing retinal thickness and CNV size over 2 years. ${ }^{13}$ Compared with the mean vision improvements observed at week 52, there was a mean 1-2-letter loss in all treatment groups in the second year following a switch to a capped quarterly PRN schedule with regular 4-weekly monitoring. ${ }^{13}$ The proportion of aflibercept $2 \mathrm{q} 8$ patients without retinal fluid on time-domain OCT images was $67.7 \%$ at week 52 and $50.1 \%$ at week 96 , reflecting a decrease in the proportion of patients without retinal fluid seen in all treatment groups through the second year. From weeks 52 to $96,14 \%-16 \%$ of aflibercept-treated patients received six injections or more for pronounced disease activity and $48 \%$ received $\leq 3$ injections. ${ }^{13,14}$

The decision to extend the aflibercept treatment interval at the end of year 1 is based on absence of macular fluid or other signs of exudation on OCT images and stable VA. The precise relationship between retinal morphology and visual outcomes in nAMD is often debated. Interestingly, a post hoc analysis found that, for all treatment groups in the VIEW studies, BCVA improved from baseline to week 52 independent of retinal fluid status at week 12, 1 month after a loading phase of three consecutive monthly injections. ${ }^{15}$ These data suggest that there may be a weak association 
between retinal fluid status at a single early time point and longer-term functional outcomes in patients treated with anti-VEGF therapy.

\section{Clinical studies of treat-and-extend aflibercept therapy}

In clinical settings, a treat-and-extend treatment algorithm with intravitreal anti-VEGF agents for retinal diseases has evolved as an effective and often-preferred approach to tailoring the dosing regimen for individual patients. ${ }^{16}$ Good visual outcomes with a decreasing burden of treatments and clinic visits have been reported with treat-and-extend aflibercept therapy for nAMD over 2-years' follow-up. ${ }^{17}$

In the ATLAS study, a prospective two-year clinical trial in the United States that investigated treat-and-extend aflibercept therapy in a small cohort of treatment-naïve nAMD patients $(n=40)$, there was a median BCVA improvement of 7.5 ETDRS letters (last observation carried forward) from baseline (median of 59.0 letters) to year 2. A treatment interval of 8 weeks or longer was being used in $71 \%$ and $75 \%$ of patients during the end of year 1 and end of year 2 visit, respectively. Epstein and Amrén reported a mean gain of 8.7 letters from baseline to month 18 in a retrospective consecutive case series of 85 elderly nAMD patients (mean age 80.1 years) switched to a treat-and-extend regimen after 1 year of fixed dosing with aflibercept at a clinic center in Sweden. ${ }^{19}$ Mean BCVA at baseline was 60.9 letters, a better starting vision than that of patients enrolled in the VIEW studies ( $\sim 54$ letters).

Mean VA improvement from baseline to month 24 was 6.0 letters, increasing from a mean VA of 61.4 letters $(\sim 20 / 60)$ at baseline to 67.4 letters $(\sim 20 / 45)$ at the 2-year visit, in a study of treat-and-extend aflibercept therapy in 136 eyes from 123 patients with nAMD completing 24 months of follow-up in routine clinical practice. ${ }^{17} \mathrm{~A}$ separate database observational study investigating maintenance phase treatand-extend anti-VEGF treatment for nAMD found that the most common interval at which disease reactivation first occurred was 8 weeks. $^{20}$ Treatment intervals greater than 12 weeks appeared to be associated with an increased risk of disease reactivation. ${ }^{20}$

\section{Experience and results from UK clinical settings: first-year outcomes}

One-year audit results from routine clinical practice in the UK support the efficacy of 8-weekly aflibercept dosing after three initial monthly injections for treatment-naïve nAMD patients, although there is some variability in reported outcomes between clinic centers, due in part to varied populations and case mix. Using a fixed-dosing regimen, the reported mean improvements in BCVA letter score from baseline through $\sim 1$ year range from 5.1 to 8.5 letters, with maintenance of vision (losing $<15$ letters) in $92 \%-96 \% .^{21-23}$

A retrospective, observational study of 1-year treatment outcomes of aflibercept in treatment-naïve patients with nAMD ( $n=52)$ reported outcomes that were similar to those observed in the VIEW studies: from baseline to 1 year, 96.2\% maintained vision, $26.9 \%$ gained 15 letters or more and mean VA improved by $8.5 \pm 13.4$ letters from mean starting VA of 56.5 letters, with a mean of $7.2 \pm 1.6$ injections. ${ }^{21} \mathrm{~A}$ retrospective multicenter data analysis by the UK Aflibercept Users Group found that the proportion of eyes achieving a VA $\geq 70$ letters ( $\geq 20 / 40$ ) doubled from $16.4 \%$ at baseline to $33.7 \%$ at 1 year in a consecutive series of treatment-naïve $n A M D$ patients ( $n=1,321$ eyes) treated with aflibercept fixed dosing. ${ }^{22}$ In the VIEW studies, $32.6 \%$ of patients receiving bimonthly aflibercept after a loading phase achieved a $\mathrm{VA} \geq 70$ letters at week 52 follow-up. ${ }^{12}$

Results from a single-center electronic medical record data analysis of 255 eyes of 223 patients with treatment-naïve nAMD receiving fixed 8-weekly aflibercept dosing after an initial loading phase showed a mean VA gain from baseline (mean VA 52 letters) to month 11 of 8 letters, with a total of seven injections given. ${ }^{23}$ Notably, the VA gain evident after the loading phase was maintained through the 1-year fixeddosing treatment plan. These efficacy outcomes are comparable to those of the VIEW studies, which reported a visual improvement of 8.4 letters from baseline to week 52 with a mean of 7.6 injections for the aflibercept $2 \mathrm{q} 8$ group. ${ }^{13}$

\section{Year 2 outcomes of aflibercept therapy for $n A M D$}

Several UK clinic centers have reported 2-year outcomes of intravitreal aflibercept therapy for nAMD. A modified capped PRN regimen (with bimonthly monitoring) and a treat-and-extend regimen in year 2 were equally effective in maintaining first-year visual improvements in a consecutive case series of treatment-naïve nAMD patients (50 eyes of 49 patients) treated at Southampton General Hospital, all with a dry macula at month 11 following aflibercept fixed dosing. ${ }^{24}$ Mean visual improvement from baseline to month 11 ranged from 6 to 8 letters, and both proactive treatment approaches through year 2 effectively stabilized the visual gains achieved in the first year.

Eleftheriadou and colleagues reported mean VA improvements from baseline (mean 55.9 letters) to year 1 and year 2 of 


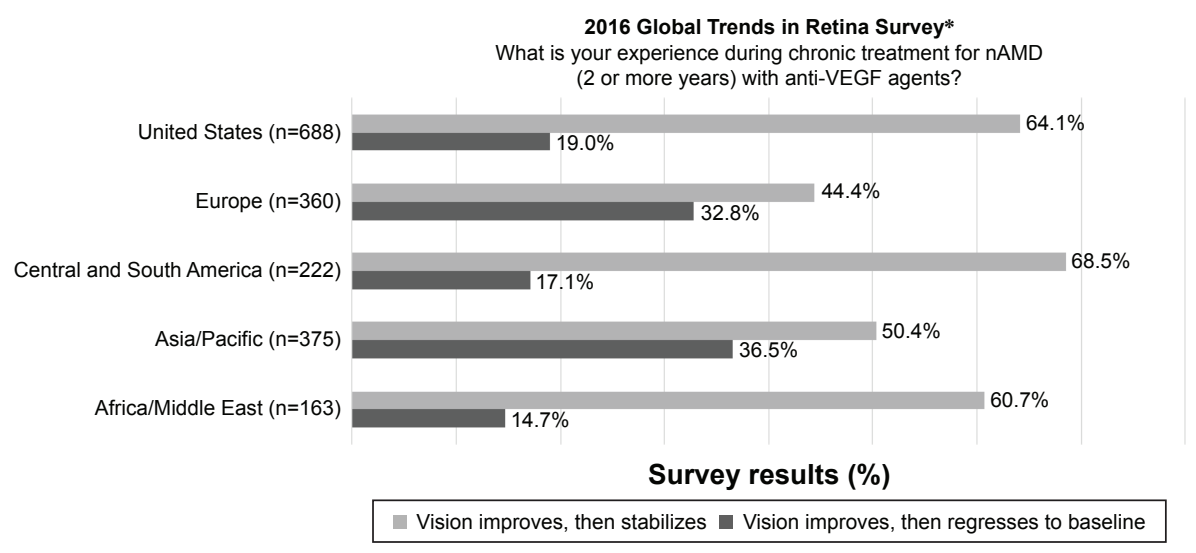

Figure 2 Experience of retina specialists of treatment responses during chronic treatment for nAMD with anti-VEGF therapy, 2016 Global Trends in Retina Survey. Notes: *Members of 39 retina societies throughout the world participated in the 2016 Global Trends in Retina Survey conducted by the American Society of Retina Specialists (ASRS). The US response category reflects US ASRS members' answers to the same question in the 2016 ASRS Preferences and Trends Survey. Reproduced with permission from the ASRS. ${ }^{27}$

Abbreviations: anti-VEGF, anti-vascular endothelial growth factor; nAMD, neovascular age-related macular degeneration.

5.4 and 5.1 letters, respectively, in a retrospective case series of previously untreated nAMD patients (109 eyes of 102 patients) treated at Moorfields Eye Hospital, London, with a mean of 11.4 injections per eye throughout the 2-year follow-up period (mean 99.3 weeks). ${ }^{25}$ In a subgroup of patients treated with aflibercept $2 \mathrm{q} 8$ in the first 12 months followed by treat-andextend retreatment in year 2 (66 eyes of 62 patients), mean VA improvement from baseline (mean 54.9 letters) to year 2 was 7.1 letters, showing vision stabilization after a mean visual improvement of 7.3 letters in year 1 . Treated with a mean of 13.5 injections, $90.4 \%$ of patients maintained vision, and the proportion of eyes with a VA $\geq 20 / 40$ increased from $13.6 \%$ at baseline to $31.8 \%$ at year 2 .

Nevertheless, there is evidence suggesting that some patients may benefit from fixed 8-weekly dosing in the second year to maintain the treatment gains achieved during the first 12 months of aflibercept therapy. A national multicenter audit of 2-year VA outcomes of nAMD patients $(n=1,083)$ treated with aflibercept showed a loss of VA improvement from year 1 to year 2 after switching from fixed bimonthly to variable dosing. ${ }^{26}$ The greatest decline was observed in eyes receiving three or fewer injections during the second year. These and other data suggest that a fixed-dosing regimen and higher treatment frequency in the second year may mitigate or reduce the risk of subtle loss of visual and anatomic improvements often noted when a variable dosing regimen is used. ${ }^{13,26}$

\section{Follow-up results through 4 years of aflibercept therapy}

While a majority of retina specialists worldwide report vision improvement then stabilization during long-term treatment of nAMD with anti-VEGF therapy, regression to baseline after initial gains is not an uncommon experience (Figure 2). ${ }^{27}$ Data capturing visual outcomes and treatment approaches with aflibercept therapy beyond 2 years are emerging, revealing maintained benefit longer term.

The VIEW 1 Extension Study involved a modified quarterly dosing schedule later amended to treatment at least every 8 weeks, with a median study duration of $\sim 116$ weeks. Enrolled patients $(n=323)$ gained a mean 7.1 letters through $\sim 4$ years' follow-up, compared with a mean improvement of 10.2 letters at week 96 for the aflibercept 2 q8 group in the randomized multicenter VIEW 1 trial. ${ }^{28}$ There was a mean loss of 2.7 letters from the VIEW 1 extension baseline through a median 116 weeks' follow-up in the extension study, and the mean number of injections was 12.9. The results demonstrate that continued aflibercept treatment using a fixed-dosing schedule through a further 2 years largely stabilized the VA gains seen during the first 96 weeks of treatment in VIEW $1 .{ }^{28}$

\section{Conclusion}

Baseline demographics and disease characteristics will vary and it is currently not possible to identify at the outset which individuals or subgroups will require more or less frequent treatments with ongoing anti-VEGF maintenance therapy. Essentially the treatment decision at the end of year 1 is whether to continue treating with fixed 8-weekly dosing or transition to a continuous variable dosing regimen for the second year.

The criteria for not extending the treatment interval reflect signs of nAMD that are associated with abnormal vessel permeability or vessel growth. Clinician experience 
suggests that nAMD patients often require regular 8-weekly dosing through the second year of aflibercept treatment, and in many ways fixed dosing offers advantages for a chronic condition, providing predictable retreatment frequencies and reducing the risk of undertreatment. Undoubtedly there will be patients who demonstrate a fluid-free macula and stabilized vision over an extended treatment interval longer than 8 weeks with a treat-and-extend approach, demonstrated by emerging data on second-year efficacy outcomes. Practitioners report achieving good results in selected patients when treatment is extended and fixed at 9-week intervals for three consecutive doses before considering further extension or shortening.

The authors hope that the 2017 updated national consensus recommendations for continued aflibercept therapy for nAMD after year 1 will be of use to clinicians when considering treatment plans to stabilize morphologic and functional improvements longer term.

\section{Acknowledgments}

The national roundtable panel meeting in 2016 that formed the basis of this 2017 update was sponsored by Bayer. All authors contributed to development and critical appraisal of draft reports presenting consensus recommendations and approved the final manuscript for publication submission. The authors, with the exception of Jackie Napier, received honoraria. Medical writing assistance was funded by Bayer and provided by Rod McNeil, BA, MBA, from Rod McNeil Associates. Bayer checked that the content was factually accurate, balanced and compliant with the Association of the British Pharmaceutical Industry Code of Practice. The views expressed are those of the author(s) and not necessarily those of Bayer.

\section{Disclosure}

Praveen J Patel: Consulting fees from Bayer, Novartis, Oxford Bioelectronics and Roche; lecture fees from Allergan; research funding from Bayer. Helen Devonport: Consulting fees from Bayer and Allergan; lecture fees from Allergan, Bayer and Novartis. Sobha Sivaprasad: Consulting fees from Allergan, Bayer, Novartis and Roche; lecture fees from Allergan, Bayer and Novartis; research funding from Allergan and Bayer. Adam H Ross: Consulting fees from Allergan and Bayer; lecture fees from Novartis. Gavin Walters: Consulting fees from Bayer; lecture fees from Bayer; research funding from Novartis, Bayer, Allergan and Alcon. Richard P Gale: Consulting fees from Novartis, Bayer and Alimera Sciences; lecture fees from Novartis and Bayer; research funding from
Bayer and Fight for Sight. Andrew J Lotery: Consulting fees and educational grants from Bayer. Sajjad Mahmood: Consulting fees from Novartis and Bayer; lecture fees from Novartis and Bayer. James S Talks: Consulting fees from Bayer and Novartis; lecture fees from Allergan and Bayer; research funding from Novartis and Bayer. Jackie Napier is employed by Bayer plc, serving as Medical Director for Ophthalmology and Neurology. The authors report no other conflicts of interest in this work.

\section{References}

1. The Royal College of Ophthalmologists. Age-related macular degeneration: guidelines for management. The Royal College of Ophthalmologists; 2013. Available from: https://www.rcophth.ac.uk/wp-content/ uploads/2014/12/2013-SCI-318-RCOphth-AMD-Guidelines-Sept2013-FINAL-2.pdf. Accessed April 21, 2017.

2. Chong V. Ranibizumab for the treatment of wet AMD: a summary of real-world studies. Eye (Lond). 2016;30(2):270-286.

3. Holz FG, Tadayoni R, Beatty S, et al. Multi-country real-life experience of anti-vascular endothelial growth factor therapy for wet age-related macular degeneration. Br J Ophthalmol. 2015;99(2):220-226.

4. Maguire MG, Martin DF, Ying GS, et al; Comparison of Agerelated Macular Degeneration Treatments Trials (CATT) Research Group. Five-year outcomes with anti-vascular endothelial growth factor treatment of neovascular age-related macular degeneration: the Comparison of Age-Related Macular Degeneration Treatments Trials. Ophthalmology. 2016;123(8):1751-1761.

5. McKibbin M, Devonport H, Gale R, et al. Aflibercept in wet AMD beyond the first year of treatment: recommendations by an expert roundtable panel. Eye (Lond). 2015;29(Suppl 1):S1-S11.

6. Freund KB, Korobelnik JF, Devenyi R, et al. Treat-and-extend regimens with anti-VEGF agents in retinal diseases: a literature review and consensus recommendations. Retina. 2015;35(8):1489-1506.

7. National Institute for Health and Care Excellence. Aflibercept solution for injection for treating wet age-related macular degeneration. Technology appraisal guidance [TA294]. National Institute for Health and Care Excellence; 2013. Available from: http://www.nice.org.uk/guidance/ ta294. Accessed April 21, 2017.

8. Scottish Medicines Consortium. Aflibercept $40 \mathrm{mg} / \mathrm{mL}$ solution for intravitreal injection (Eylea ${ }^{\circledR}$ ) SMC No. (857/13); 2013. Available from: www.scottishmedicines.org.uk. Accessed April 21, 2017.

9. Lucentis ${ }^{\circledR}$ (ranibizumab solution for injection) [summary of product characteristics]. Camberley, UK: Novartis Europharm Ltd; 2016. Available from: https://www.medicines.org.uk/emc/medicine/19409. Accessed April 21, 2017.

10. Eylea ${ }^{\circledR}$ (aflibercept solution for injection) [summary of product characteristics]. Leverkusen, Germany: Bayer AG; 2017. Available from: https://www.medicines.org.uk/emc/medicine/27224. Accessed August 9, 2017.

11. Age-Related Eye Disease Study Research Group. A randomized, placebo-controlled, clinical trial of high-dose supplementation with vitamins $\mathrm{C}$ and $\mathrm{E}$, beta carotene, and zinc for age-related macular degeneration and vision loss: AREDS report no. 8. Arch Ophthalmol. 2001; 119(10):1417-1436.

12. Heier JS, Brown DM, Chong V, et al; VIEW 1 and VIEW 2 Study Groups. Intravitreal aflibercept (VEGF trap-eye) in wet age-related macular degeneration. Ophthalmology. 2012;119(12):2537-2548.

13. Schmidt-Erfurth U, Kaiser PK, Korobelnik JF, et al. Intravitreal aflibercept injection for neovascular age-related macular degeneration: ninety-six-week results of the VIEW studies. Ophthalmology. 2014; 121(1):193-201.

14. Richard G, Monés J, Wolf S, et al. Scheduled versus pro re nata dosing in the VIEW trials. Ophthalmology. 2015;122(12):2497-2503. 
15. Moshfeghi DM, Hariprasad SM, Marx JL, et al. Effect of fluid status at week 12 on visual and anatomic outcomes at week 52 in the VIEW 1 and 2 trials. Ophthalmic Surg Lasers Imaging Retina. 2016;47(3): 238-244.

16. Stone TW, Raef S, editors. American Society of Retina Specialists 2016 Global Preferences and Trends Membership Survey. Presentation at: The 2016 Annual Meeting of the American Academy of Ophthalmology; October 14-18; 2016; Chicago, IL.

17. Barthelmes D, Nguyen V, Daien V, et al; Fight Retinal Blindness Study Group. Two year outcomes of "treat and extend" intravitreal therapy using aflibercept preferentially for neovascular age-related macular degeneration. Retina. 2017. Epub ahead of print.

18. DeCroos FC, Reed D, Adam MK, et al. Treat-and-extend therapy using aflibercept for neovascular age-related macular degeneration: a prospective clinical trial. Am J Ophthalmol. 2017. Epub ahead of print.

19. Epstein D, Amrén U. Near vision outcome in patients with age-related macular degeneration treated with aflibercept. Retina. 2016;36(9): 1773-1777.

20. Essex RW, Nguyen V, Walton R, et al; Fight Retinal Blindness Study Group. Treatment patterns and visual outcomes during the maintenance phase of treat-and-extend therapy for age-related macular degeneration. Ophthalmology. 2016;123(11):2393-2400.

21. Vazquez-Alfageme C, Patel PJ, Hamilton R. Efficacy of aflibercept in treatment-naïve neovascular age-related macular degeneration: one-year real world outcomes. Presentation at: The Association for Research in Vision and Ophthalmology (ARVO) 2015 Annual Meeting; May 3-7; 2015; Denver, CO.
22. Talks JS, Lotery AJ, Ghanchi F, et al; United Kingdom Aflibercept Users Group. First-year visual acuity outcomes of providing aflibercept according to the VIEW study protocol for age-related macular degeneration. Ophthalmology. 2016;123(2):337-343.

23. Almuhtaseb H, Kanavati S, Rufai SR, Lotery AJ. One-year real-world outcomes in patients receiving fixed-dosing aflibercept for neovascular age-related macular degeneration. Eye (Lond). 2017;31(6): 878-883.

24. Almuhtaseb H, Rennie C, Sahu D, Lotery A. Aflibercept 2nd year outcomes in nAMD: unicenter data analysis (Capped PRN vs. Treat and Extend). Presentation at: The 16th European Society of Retina Specialists (EURETINA) Congress; September 8-11; 2016; Copenhagen, Denmark.

25. Eleftheriadou M, Vazquez-Alfageme C, Citu CM, et al. Long-term outcomes of aflibercept treatment for neovascular age-related macular degeneration in a clinical setting. Am J Ophthalmol. 2017;174: $160-168$.

26. Almuhtaseb H, Johnston RL, Talks JS, Lotery AJ. Second-year visual acuity outcomes of nAMD patients treated with aflibercept: data analysis from the UK Aflibercept Users Group. Eye (Lond). 2017. Epub ahead of print.

27. Rezaei KA, Stone TW, editors. 2016 Global Trends in Retina Survey. Chicago, IL: American Society of Retina Specialists; 2016.

28. Kaiser PK, Singer M, Tolentino M, et al. Long-term safety and visual outcome of intravitreal aflibercept in neovascular age-related macular degeneration. VIEW 1 Extension Study. Ophthalmol Retina. 2017;1(4); 304-313.
Clinical Ophthalmology

\section{Publish your work in this journal}

Clinical Ophthalmology is an international, peer-reviewed journal covering all subspecialties within ophthalmology. Key topics include: Optometry; Visual science; Pharmacology and drug therapy in eye diseases; Basic Sciences; Primary and Secondary eye care; Patient Safety and Quality of Care Improvements. This journal is indexed on Submit your manuscript here: http://www.dovepress.com/clinical-ophthalmology-journal

\section{Dovepress}

PubMed Central and CAS, and is the official journal of The Society of Clinical Ophthalmology (SCO). The manuscript management system is completely online and includes a very quick and fair peer-review system, which is all easy to use. Visit http://www.dovepress.com/ testimonials.php to read real quotes from published authors. 